\title{
Nanoscale
}

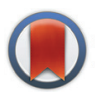

CrossMark $\leftarrow$ click for updates

Cite this: Nanoscale, 2016, 8, 987

Received 25th September 2015 Accepted 24th November 2015

DOI: 10.1039/c5nr06657c

www.rsc.org/nanoscale

\section{Nanostar probes for tip-enhanced spectroscopy $\dagger$}

\author{
Woong Kim, $\star^{a}$ Nara Kim, $t^{b}$ Joon Won Park ${ }^{a}$ and Zee Hwan Kimb
}

\section{Introduction}

Tip-enhanced near-field optical microscopy (TENOM) $)^{1-6}$ and super-resolution fluorescence microscopy ${ }^{7,8}$ constitute two optical imaging techniques providing not only nanometric spatial resolution ${ }^{9}$ but also key molecular structural information of individual molecules ${ }^{10,11}$ on the sample's surface. Such information ultimately helps establish the correlation between chemical structure and functionality at the nanometer scale. ${ }^{12-15}$ Super-resolution fluorescence microscopy, such as stimulated emission depletion (STED) microscopy, stochastic optical reconstruction microscopy (STORM) and photoactivated localization microscopy (PALM), critically relies on the photophysics of the dyes (photostability, excited state lifetime, and quantum yield). On the other hand, TENOM can be applied to a wider range of fluorescent and non-fluorescent molecules but is critically dependent on the local field enhancement at the tip. ${ }^{16}$

A key factor in the TENOM probe design is to enhance the electric field at the probe apex upon laser illumination. It was observed that the lateral resolution and the local field

\footnotetext{
${ }^{a}$ Department of Chemistry, Pohang University of Science and Technology, San 31 Hyoja-dong, Pohang, 790-784, Korea.E-mail: jwpark@postech.ac.kr ${ }^{b}$ Department of Chemistry, Seoul National University, Seoul, 151-742, Korea. E-mail: zhkim@snu.ac.kr

$\dagger$ Electronic supplementary information (ESI) available: Illustrations of TERS and TEF experiments, information about the TEM images, scheme of surface preparation and peak assignments of TERS spectra. See DOI: 10.1039/c5nr06657c $\$$ These authors contributed equally to this work.
}

enhancement critically depend on the geometry and composition of the probes. TENOM researchers have reported various forms of probes and their preparation methods, such as e-beam coating of $\mathrm{Ag}$ and $\mathrm{Au}$ on dielectric probes, ${ }^{17,18}$ chemical etching of silver and gold wires, ${ }^{19,20}$ FIB milling of probes and fibers, ${ }^{21-23}$ and attaching nanoparticles to the apexes of fiber probes. ${ }^{24-26}$ Among them, the nanoparticle-tip appears attractive because we may be able to synthetically fine-tune the shape and composition of the attached nanoparticles for better plasmonic field enhancement. For example, if the tip could be functionalized with an octahedron ${ }^{27}$ or nanostar ${ }^{28-30}$ made of silver or gold, one may be able to maximize the enhancement and improve the spatial resolution. The sharp edges of such nanocrystals result in a stronger electromagnetic field than spherical nanoparticles, as confirmed by surfaceenhanced Raman scattering and surface-enhanced fluorescence measurements. ${ }^{31}$ Furthermore, for tip-enhanced fluorescence (TEF), it was found that the isolated structures (such as nanospheres or truncated cones) are essential to maintain sufficient field enhancement. ${ }^{32,33}$ In this regard, a nanostar particle probe is one of the most promising candidates to be placed on a tip because the spikes of nanostars are expected to form effective electromagnetic hot spots.

In this article, we report the fabrication procedure of a nanostar probe for tip-enhanced spectroscopy. Fabrication of the tip involves the transfer of a single gold nanoparticle (AuNP, $5 \mathrm{~nm}$ ) seed from the substrate to the tip through the differential binding forces of DNA-DNA hybridization, ${ }^{34-36}$ and it involves the reductive deposition of metals to form a nanostar placed at the end of the scanning probe. We observed 
that such tips showed appreciable field enhancement for use in tip-enhanced near-field spectroscopy.

\section{Methods}

\section{General}

The silane coupling agent $\mathrm{N}$-(3-(triethoxysilyl)-propyl)-O-polyethyleneoxide urethane (TPU) was purchased from Gelest. Gold nanoparticle seeds $(5 \mathrm{~nm})$ were purchased from Ted Pella. Oligonucleotides were purchased from IDT. The atomic force microscopy probes employed for the picking process were purchased from BudgetSensors. Contact mode (ContGD-G) and tapping mode (Tap190-G) probes were used for TERS and TEF, respectively. Probes for the control experiment were e-beam coated with $\mathrm{Ti}(1 \mathrm{~nm})$ and $\mathrm{Au}(10 \mathrm{~nm})$ as an adhesion layer. Preparation of dendron-modified probes and substrates were reported, ${ }^{37}$ and now they are commercially available through NSB POSTECH Inc. The pick-up procedure employed is similar to that described in previous reports. ${ }^{34,35}$ Clean cover glasses were coated with $\mathrm{Au}$ (10 nm) using an e-beam evaporator for the TERS experiment. Freshly prepared gold film on a cover glass was used to measure Raman enhancement. A stock solution of crystal violet was diluted in ethanol, and a drop of $10 \mu \mathrm{L}$ was placed on the gold-coated cover glasses. Transmission electron microscopy (TEM, JEM-1011, JEOL) was used to image the tips.

\section{Chemicals}

$\mathrm{HAuCl}_{4}$ (99.999\%, Sigma Aldrich), silver nitrate (99.9999\%, Sigma Aldrich), and ascorbic acid ( $\geq 99.0 \%$, Sigma Aldrich) were used to synthesize the nanostars. All reagent stock solutions were prepared with ultrapure deionized water. DiI stock solution for TEF was prepared in DMSO and diluted in toluene before its use. The quantum yield of 1,1'-dioctadecyl-3,3,3',3'tetramethylindocarbocyanine perchlorate (DiI, Sigma-Aldrich, $\left.\lambda_{\max }=549 \mathrm{~nm}\right)$ is 0.07 in methanol. Crystal violet $(\geq 90.0 \%$, Sigma Aldrich) for TERS was dissolved in ethanol.

\section{TENOM set-up}

The TENOM set-up consisted of an inverted microscope (IX71, Olympus), a Raman spectrometer (Triax 320, Horiba; DU-401, Andor Tech), avalanche photodiodes (APDs), and an atomic force microscope (AFM, XE-120, Park Systems) operating under contact and tapping modes. The laser-beam (wavelength of $532 \mathrm{~nm}, \mathrm{Nd}$ :YAG) was focused onto the tip through an objective lens (oil immersion, NA $=1.46$ ), and Raman and fluorescence signals were collected through the same objective lens (see ESI Fig. S1†).

\section{Preparation of nanostar attached probes}

Nanostar probes were prepared by attaching a Au nanoparticle to the probe and proceeding with the growth process. Twenty microliters of $50 \mathrm{mM} \mathrm{HAuCl}$ and the same volume of $1.0 \mathrm{M}$ $\mathrm{HCl}$ were added to $20 \mathrm{~mL}$ deionized pure water. Under vigorous stirring, $40 \mu \mathrm{L}$ of $2.0 \mathrm{mM} \mathrm{AgNO}_{3}$ solution and $20 \mu \mathrm{L}$ of
$100 \mathrm{mM}$ freshly prepared ascorbic acid solution were added. The probes were added into the solution immediately after the mixing. The yellowish color of the resulting solution changed to green after a few minutes. After the color changed to green, the immersed probes were rinsed by deionized water to remove nonspecifically bound particles and excess chemicals. The resulting nanostar probes were characterized by TEM.

\section{Spin coating of PMMA}

To make a PMMA layer less than $2 \mathrm{~nm}$ thick on a cover glass, a toluene solution (1 mL) of PMMA (15000 MW, $0.50 \mathrm{mg}$ ) and DiI $\left(10^{-8} \mathrm{M}\right)$ was prepared. A drop of the solution $(20 \mu \mathrm{L})$ was placed on a cover glass that had been cleaned with Piranha, RCAI and RCAII solutions. With a spin coater (Spin-1200, MIDAS System) operating at $3000 \mathrm{rpm}$, a thin layer of PMMA was formed by spinning for 60 seconds. Thickness of the PMMA layer was confirmed by scratching the surface and scanning with AFM.

\section{Results}

\section{Synthesis and optical properties of nanostars}

We first examined the plasmonic properties of nanostars in a colloid. Nanostars were synthesized by enhancing small seed Au nanoparticles (AuNP, diameter of $5 \mathrm{~nm}$, Ted Pella) and subsequently forming sharp spikes on the enlarged nanoparticles (Fig. 1A). For the enhancement, the seed solution $(100 \mu \mathrm{L}$, $65 \mathrm{nM})$ and a solution of $\mathrm{HAuCl}_{4}(200 \mu \mathrm{L}, 50 \mathrm{mM})$ was mixed with $10 \mathrm{~mL}$ of deionized water. Next, an ascorbic acid solution (200 $\mu \mathrm{L}, 100 \mathrm{mM}$ ) was added. The resulting solution was stirred for 1-2 minutes, and the color turned from yellow to pink. The seed AuNPs with a diameter of $5 \mathrm{~nm}$ (Fig. 1B) showed dipolar plasmon resonance at $\lambda=519 \mathrm{~nm}$ (Fig. 1C, black curve for scattering spectrum). The size of the seed NP increased through the reduction of gold ions to form larger NPs with a diameter of $70 \mathrm{~nm}$ (Fig. 1B). The enlarged NPs showed a slightly red-shifted resonance at $\lambda=543 \mathrm{~nm}$ (Fig. 1C, blue curve). Finally, the spikes were grown on top of the NPs by the reduction of gold on silver deposition sites as reported previously $^{30}$ (Fig. 1B, see also Fig. S2 of the ESI $\dagger$ ). The NPS with the spikes resulted in a resonance at $\lambda=809 \mathrm{~nm}$.

Such a red-shift following spike enhancement is caused by the excitation of longitudinal plasmon modes in the spikes of the nanostars. The change in scattering spectra can be fully simulated by finite-difference time-domain (FDTD) calculations, ${ }^{38,39}$ as shown in Fig. 1D. In particular, the simulated field distribution around the nanostar indicates drastically enhanced local field intensities compared with those of spherical NPs (diameter of $70 \mathrm{~nm}$ ) (Fig. 1E). The experimental spectra and the simulation demonstrate that nanostars will provide strong field enhancement that is significantly larger than that of spherical NPs.

Synthesis of nanostars on the end of a scanning probe is similar to that of the colloidal nanostars described above. In this procedure, we first attach the seed NP to the tip via DNA- 
(A)

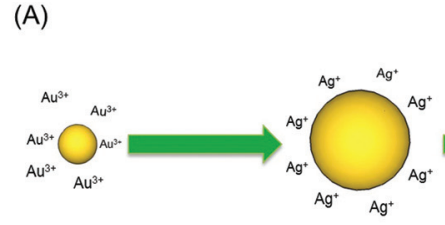

$5 \mathrm{~nm}$ AuNP
Enhanced 70nm AuNP

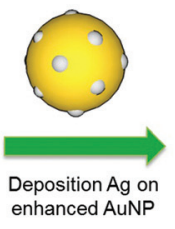
enhanced AuNP

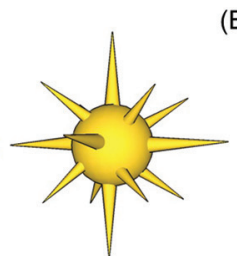

Nanostar
(B)

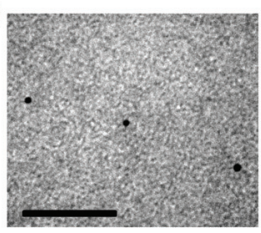

$5 \mathrm{~nm}$ AuNP

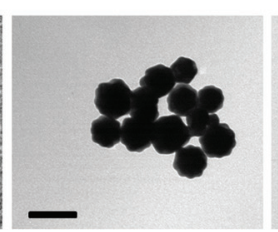

Enhanced $70 \mathrm{~nm}$ AuNP

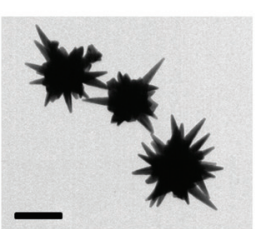

Nanostar
(C) Experiment

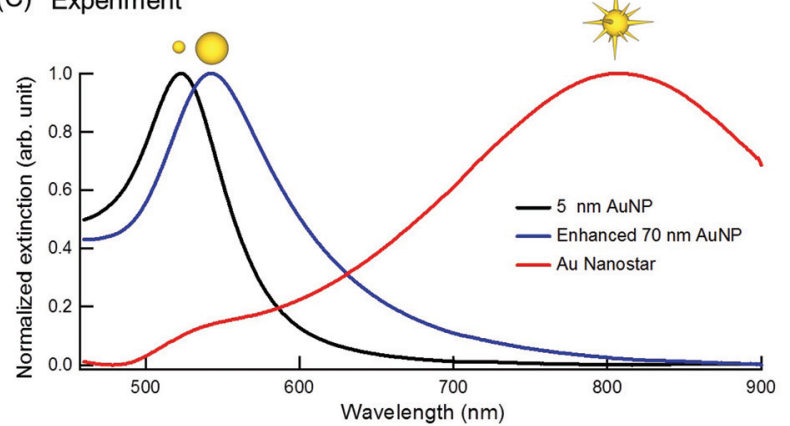

(D) Simulation

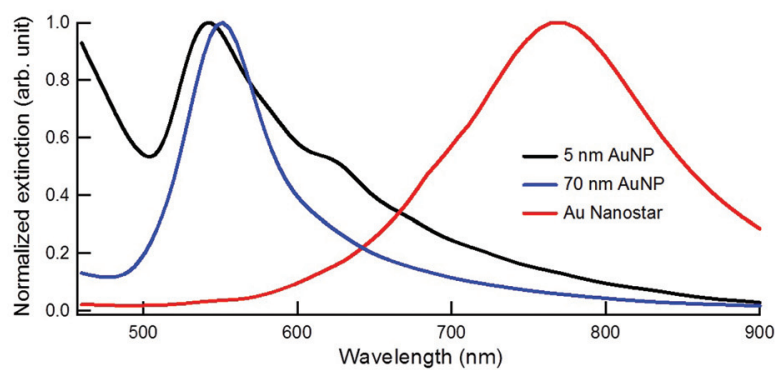

(E)
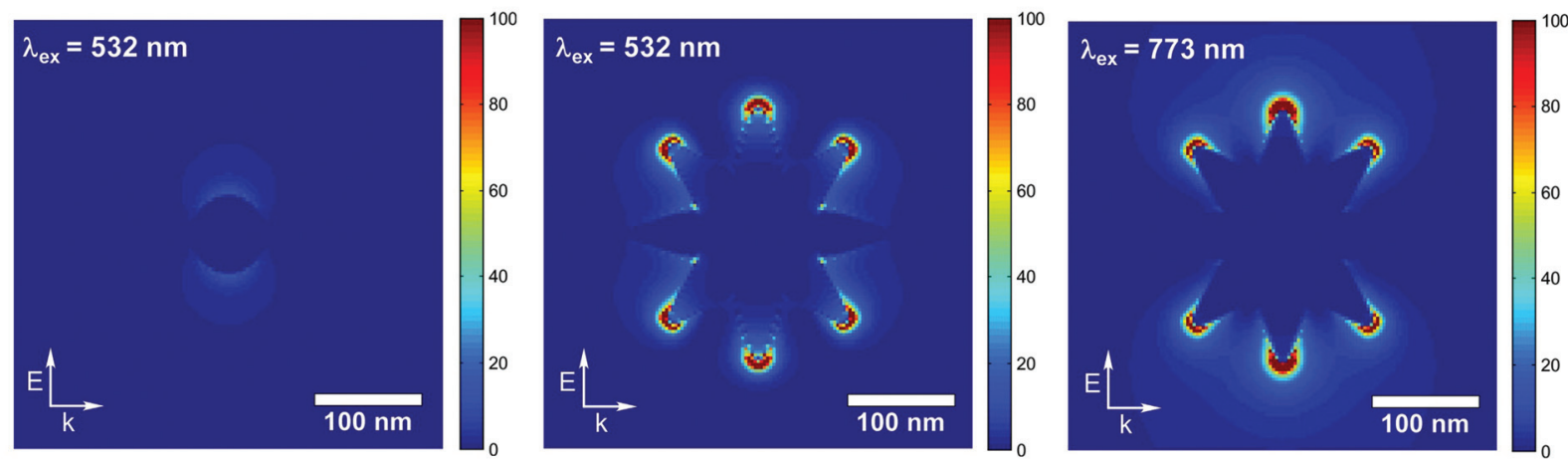

Fig. 1 Plasmonic properties of Au nanostars. (A) A schematic diagram of nanostar synthesis. (B) TEM images of $5 \mathrm{~nm}$ AuNP, enhanced $70 \mathrm{~nm}$ AuNP, and nanostars (scale bar: $100 \mathrm{~nm}$ ). (C) Extinction spectra of $5 \mathrm{~nm}$ AuNP (black), $70 \mathrm{~nm}$ AuNP (blue), and nanostars (red). The maximum absorbances of $5 \mathrm{~nm}$ AuNP, $70 \mathrm{~nm}$ AuNP, and nanostars occur at $519 \mathrm{~nm}, 543 \mathrm{~nm}$, and $809 \mathrm{~nm}$, respectively. (D) FDTD-simulated scattering spectra of the corresponding nanoparticles. (E) Simulated local field distribution around a $70 \mathrm{~nm}$ spherical AuNP at $\lambda=532 \mathrm{~nm}$, a Au nanostar at $\lambda=532 \mathrm{~nm}$, and a Au nanostar at $\lambda=773 \mathrm{~nm}$. The color-scale for intensity is expressed in $|E|^{2}$. The $E, k$ and the corresponding arrows represent the polarization and propagation directions of the incident light.

hybridization, ${ }^{34,40}$ and then grow the nanostars in situ. The alternative strategy of directly attaching fully grown nanostars to the tip (either by electrostatic pickup or by chemical functionalization) is unfeasible because the contact area between the nanostar and the tip is too small to provide sufficient adhesion between the nanoparticle and the tip.

Fig. 2A shows the transfer process of a single AuNP through the differential binding forces of DNA-DNA interactions. The AuNPs tethering a single DNA strand (63 bases) for each were hybridized onto complementary DNA with 20 bases that was immobilized onto the silicon wafer surface. ${ }^{34}$ An atomic force microscopy (AFM) tip (contact mode tip for TERS, tapping mode tip for TEF, BudgetSensors) tethering 40-base DNA approached the substrate. The single strand of DNA (ss-DNA) on the tip had a base sequence that is complementary to the single strand part of DNA attached to the $5 \mathrm{~nm}$ AuNP. Upon the tip-sample contact, the 40 base DNA on the tip hybridized with the complementary part of the DNA on the substrate. The binding force between these two strands is stronger than the force between the substrate DNA and the AuNP-bound DNA, so the ss-DNA and its bound AuNP was transferred from the substrate to the tip during retraction. In this work, the surfaces of the tip and the substrate were modified with dendrons (Fig. 2B) that serve as lateral spacers between the neighboring DNA strands. As reported previously, ${ }^{37}$ the dendron-modified AFM tip guarantees single molecular interaction (in our case, transfer of a single seed NP). ${ }^{41}$ Using the procedure described above, we transferred AuNP seeds to the tips with a success rate of $70 \%$ (ESI, Fig. S3†). The TEM image in Fig. 2C shows the transferred AuNP seed.

Fig. 3 schematically shows the one-pot synthetic procedure for fabricating a nanostar probe, which includes the growth of the NP (Fig. 3A, second step) and the subsequent growth of sharp spikes on the NP (Fig. 3B, third step). The procedure we employed is based on the method by Yuan et al., ${ }^{30}$ but was modified to avoid nonspecific growth. More typical procedures 
(A)

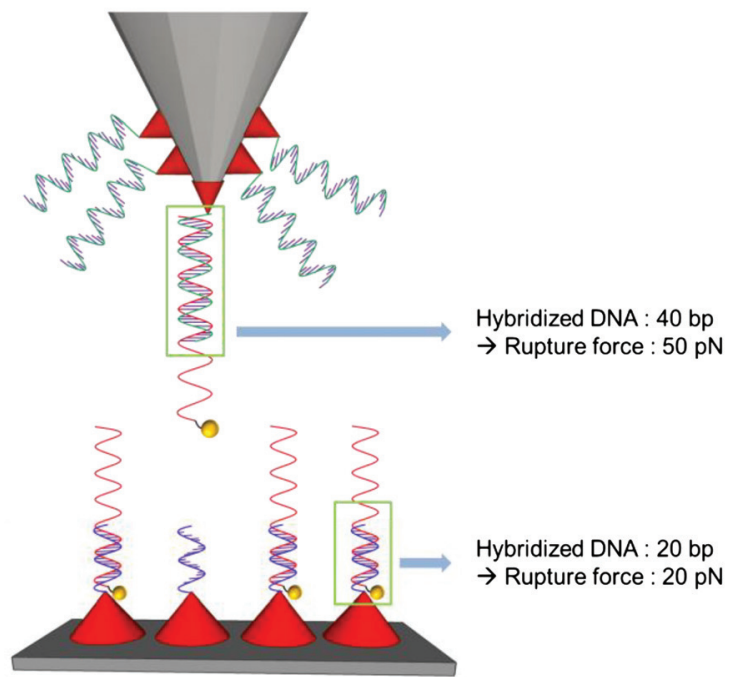

(B)

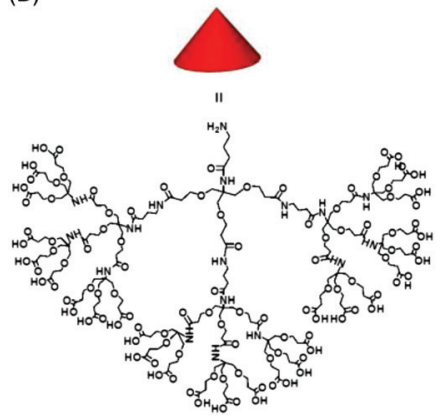

(C)

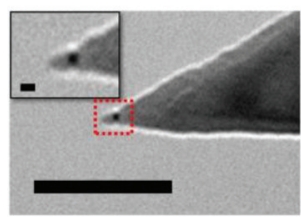

Fig. 2 The first step in making nanostar probes. (A) A schematic illustrating the transfer from the substrate to the tip of a single DNA strand tethering a AuNP. (B) Chemical structure of the dendron used for the surface modification. (C) TEM image of a tip with a single AuNP transferred from the substrate (scale bar: $100 \mathrm{~nm}$ ). The inset shows the magnified view of the single AuNP (scale bar: $10 \mathrm{~nm}$ ).

of nanostar generation, which involve the use of shapedirecting reagents such as cetyltrimethylammonium bromide $(\mathrm{CTAB})^{46}$ or polyvinylpyrrolidone (PVP), ${ }^{47}$ were not employed

because such reagents tend to generate background signals in TERS and TEF measurements.

In our procedure, the seeded tip was immersed in the "developing" solution ${ }^{30}$ for 1-2 minutes. The developing solution was prepared by first mixing $\mathrm{HAuCl}_{4}(50 \mu \mathrm{M})$ and $\mathrm{AgNO}_{3}$ $(4 \mu \mathrm{M})$ solutions and then adding ascorbic acid solution $(100 \mu \mathrm{M})$ immediately after mixing. The developing solution both enhanced the size of seed NP (second step) and produced the spikes on the enhanced NP (third step). In the second step (Fig. 3A), the seed NP was grown into a larger NP with a diameter of $\sim 70 \mathrm{~nm}$. For this growth step, ascorbic acid reduced $\mathrm{Au}^{3+}$ preferentially in the developing solution because $\mathrm{Au}$ ions have a higher reducing potential than $\mathrm{Ag}$ ions $\left(\mathrm{Au}^{3+}+3 \mathrm{e}^{-} \rightarrow\right.$ $\mathrm{Au}, 1.5 \mathrm{eV} ; \mathrm{Ag}^{+}+\mathrm{e}^{-} \rightarrow \mathrm{Ag}, 0.8 \mathrm{eV}$ ). In the third step (Fig. 3B), small $\mathrm{Ag}$ islands were deposited onto the enhanced NP through the under-potential reduction and deposition ${ }^{42-45}$ of silver ions by ascorbic acid. The silver islands on the enhanced NP served as active sites on the gold surface at which the gold ions were efficiently reduced to form gold spikes. It is known that the surface density of silver islands determines the number and shape of the spikes. A careful tuning of $\mathrm{Ag}^{+}$concentration is important for the generation of spikes with the desired dimensions. Solutions with high $\mathrm{Ag}^{+}$concentrations $(>40 \mu \mathrm{M})$ led to very short spikes (length: 15-20 nm) and significant nonspecific growth on the tip body. Solutions of low concentration $(<0.04 \mu \mathrm{M})$ led to no spikes on the surface of AuNP. We found that the medium concentration $(4.0 \mu \mathrm{M})$ yielded nanostars with sufficiently long spikes (length = 50-80 nm), while the number of spikes was smaller than that of nanostars grown in solution (Fig. S2 $\dagger$ ). Additionally, we observed that a solution with high $\mathrm{Au}^{3+}$ concentration induced nonspecific formation of nanoparticles on the whole body of the AFM tip, and slow stirring resulted in nanostars with toothin branches. The use of a dilute Au solution $(50 \mu \mathrm{M})$ suppressed the nonspecific growth of unwanted parts and a stirring speed of 700-800 rpm resulted in spikes with sufficient thickness $(\sim 10 \mathrm{~nm})$.

Fig. 4 displays three representative TEM images of the fabricated nanostar probes in which the spikes with a radius of $<10 \mathrm{~nm}$ are located at the apex of the tip. Typically, ten out of

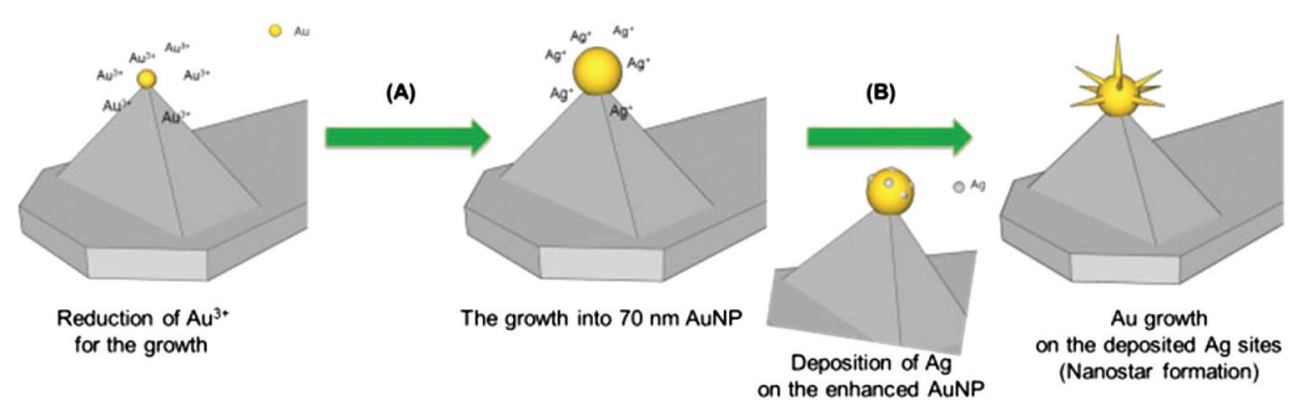

Fig. 3 A schematic for the preparation of the nanostar probe by enhancing a seed AuNP picked up by an AFM tip. (A) Through the picking process, a single AuNP of $5 \mathrm{~nm}$ was attached to the end of a tip. The tip was immersed in a solution of gold ions, silver ions, and a reductant. Gold ions were reduced to make the initial nanoparticle bigger. (B) Tiny silver particles were deposited on the enhanced gold surface, and the silver deposits initiated formation of gold spikes. The resulting nanostar sits on top of the probe. 

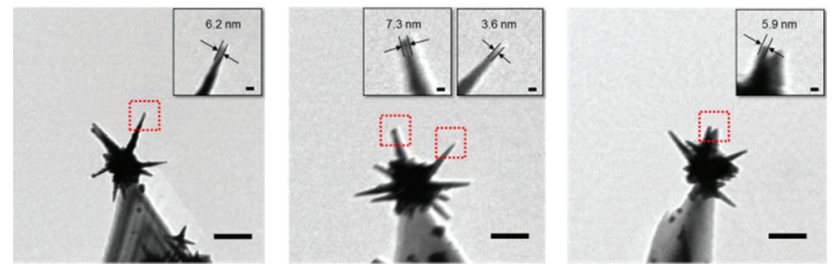

Fig. 4 TEM images of select nanostar probes (scale bars: $100 \mathrm{~nm}$ ). The insets show a magnified view of the spikes (scale bars: $10 \mathrm{~nm}$ ).

15 tips formed a nanostar at the tip apex (yield $=67 \%$ ). We found that the orientation (relative angle between the axes of the tips and the spikes) of each spike was hard to control. The nanostar tips were found to have mechanical stabilities sufficient for AFM scanning. In only one or two cases out of ten, the nanostar was detached from the probe during scanning, although the TEM images obtained before and after the scanning show slight blunting and wear on the remaining nanostar.

Regarding the performance of the nanostar tip for the topographic measurement, we can see both its advantage and disadvantage over the typical Si-tip. For the samples of small topographic contrast (for example, single molecules, graphene, or DNA chains on a flat substrate), only one spike (usually with a radius of $<5 \mathrm{~nm}$ ) on the nanostar is expected to interact with the sample, and thus the tip will provide a spatial resolution that is equal to, or better than, the one obtained from the Si-tip. For the samples of large topographic contrast (for example, nanoparticles with $>10 \mathrm{~nm}$ diameter), several differently oriented spikes on the nanostar may interact with the nanostructure concomitantly, leading to a noticeable topographic convolution effect (ESI, Fig. S4†). In this regard, the nanostar tip is better suited to the former samples.

\section{TENOM measurement}

TERS and TEF performance of the nanostar probes were tested using a set-up consisting of an atomic force microscope and a confocal microscope with an excitation laser wavelength of $532 \mathrm{~nm}$ (ESI, Fig. S1 $\dagger$ ). A radially polarized ${ }^{48,49}$ laser beam was focused at the tip through a high-NA objective lens (ESI, Fig. $\mathrm{S} 1 \dagger$ ), and the emitted photons were collected by the same objective lens and sent to an avalanche photodiode (APD) and a Raman spectrometer.

The sample used for TERS measurement was crystal violet (CV) that was dispersed on a gold $(10 \mathrm{~nm}) / \mathrm{Ti}(2 \mathrm{~nm}) /$ glass substrate. With regard to the two consecutive TERS spectra, less than $20 \%$ of decrease in the TERS signal was observed under exposure to $80 \mu \mathrm{W}$ of laser power (ESI, Fig. S5†). Fig. 5 shows Raman spectra of the CV sample collected with and without the tip. The spectra demonstrate the pronounced enhancement of eight major peaks of CV (see ESI, Table S1† for the peak assignments). For the particular spectra shown, the ratio of peak intensities with $(I)$ and without $\left(I_{0}\right)$ the tip is $\sim 10$ as determined by the area of the peak at $804 \mathrm{~cm}^{-1}\left(I / I_{0}=215 / 20\right)$.

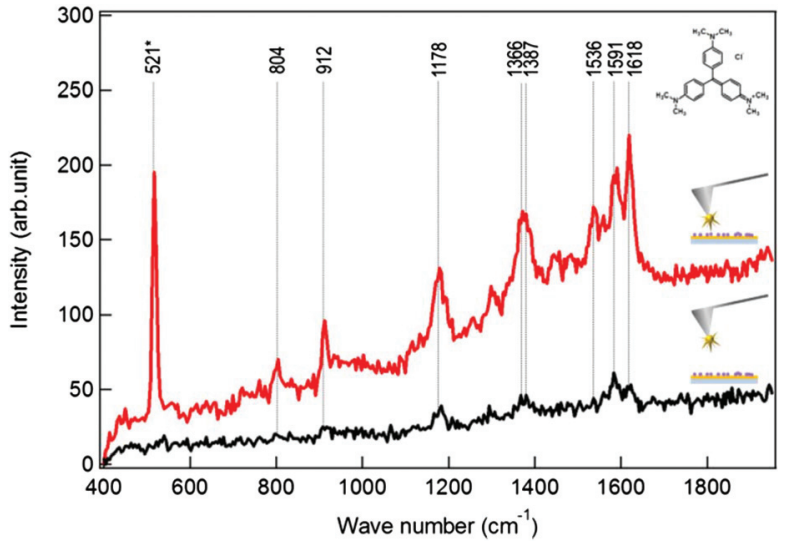

Fig. 5 TERS spectra of crystal violet (CV, inset in figure) with (red) and without (black) the nanostar tip. Laser power and exposure time were $80 \mu \mathrm{W}$ and $10 \mathrm{~s}$, respectively. Major vibrational modes of CV are indicated with vertical lines and wavenumbers. The asterisk $\left(^{*}\right)$ points to the Si-phonon mode of the tip.

Note that $I_{0}$ also contains a SERS signal of CV arising from the hotspots on $\mathrm{Au}$ surfaces. We found that the ratio varied from 2 to 10 for different tips, yet all of the tips showed TERS activity.

For TEF measurements, DiI (1,1'-dioctadecyl-3,3,3',3'-tetramethylindocarbocyanine perchlorate $)^{50}$ embedded in a PMMA layer with a thickness of $<2 \mathrm{~nm}$ was employed. The PMMA matrix served not only to immobilize but also to enhance the photostability of the DiI dye. Absorption wavelength of DiI is resonant (maximum excitation/emission wavelengths of DiI dye are 549 and $565 \mathrm{~nm}$, respectively) with the wavelength of the excitation laser (532 $\mathrm{nm}$ ). In the experiment, the laser power was controlled to less than $200 \mathrm{nW}$ in order to avoid photo-bleaching. Fig. 6A-E compare TEF images of the DiI sample with the tip engaged with the surface (Fig. 6; first row, tip-on) and with images with the tip retracted (Fig. 6; second row, tip-off). Two features are evident from the comparison. First, the tip-on images show significantly enhanced signal intensity and spatial resolution of 90-200 nm compared with the tip-off images showing diffraction-limited spot sizes of 300-400 nm. During the TEF scanning, the spikes on the nanostar probe often became blunt, possibly due to mechanical deformation by the tapping mode feedback with the sample surface. Nevertheless, the nanostar probes maintained a constant level of enhancement during several hours of usage. Second, we found that the degree of the enhancement varied from one molecule to another, with the observed optical enhancement varying from 5 times to $10^{2}$ times within the same sample. For example, only one spot out of the two in the images in Fig. 6D shows significant tip-enhancement (the lower bound for enhancement is 98 as determined by the Gaussian fitting of the line-profiles of the same TEF spot with and without the tip). Such variation may originate from different orientations of DiI molecules ${ }^{24,51,52}$ and the variability in the tip-molecule distance. Certainly, the nanostar tip produced images of individual DiI spots as the superposition 


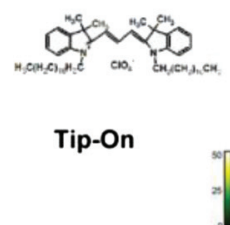

(A)
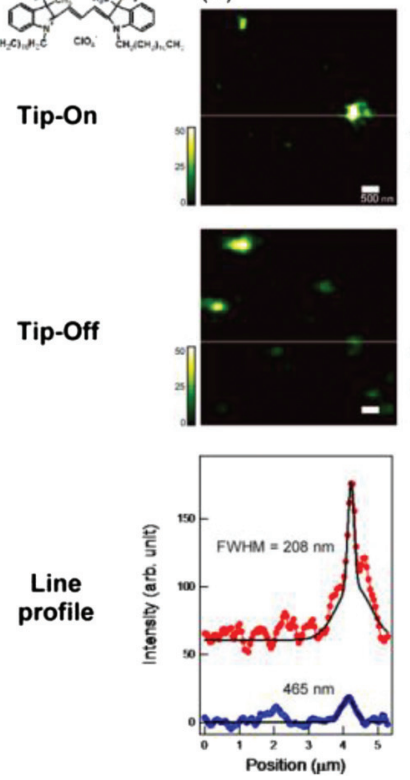

(B)
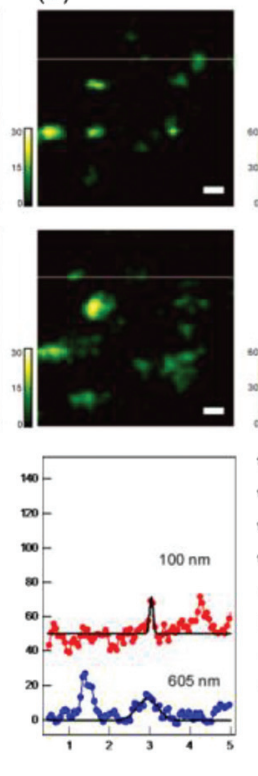

(C)
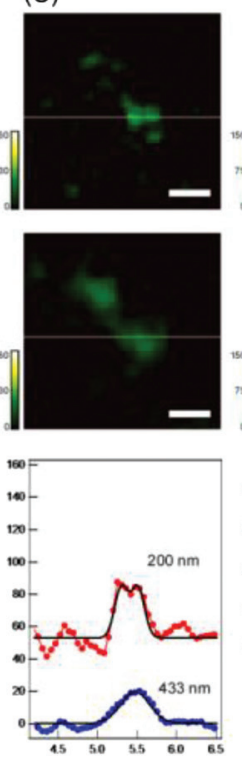

(D)
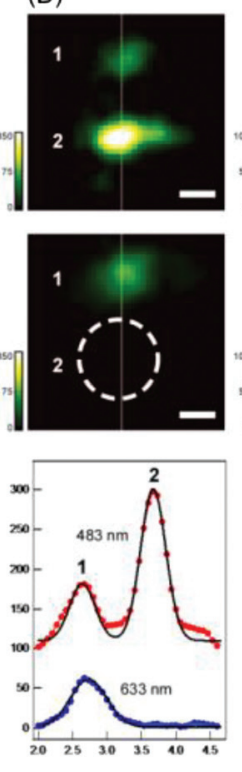

(E)
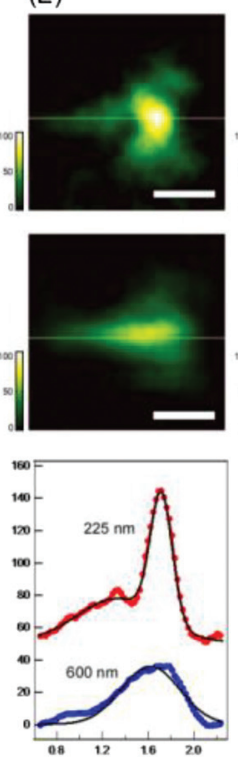

(F)
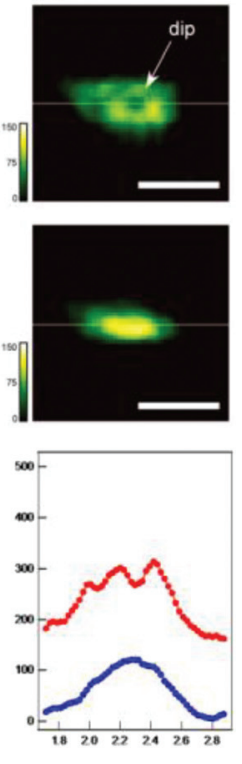

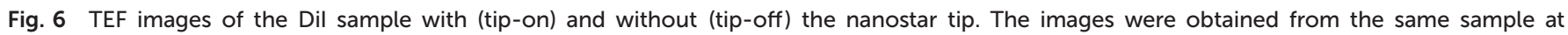

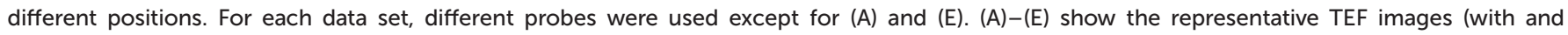

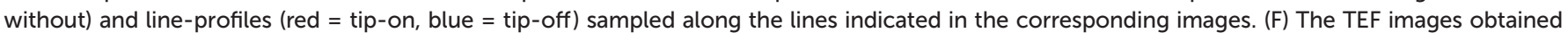

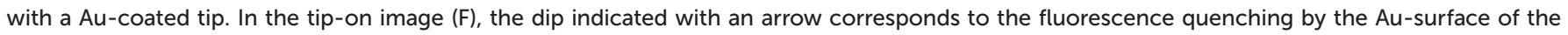

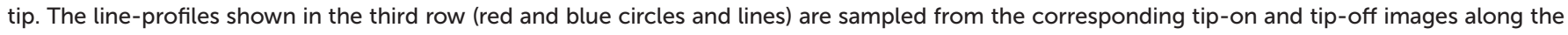

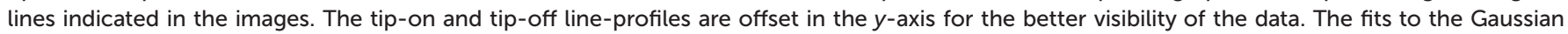

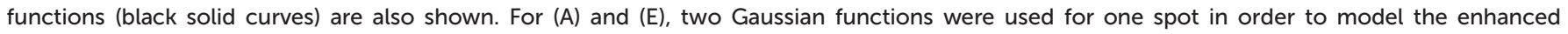
(narrow) and unenhanced (broad) components of fluorescence from the Dil spot. The scale bars in the images correspond to 500 nm.

of a diffraction-limited Gaussian point-spread function (psf) with a sharp, tip-enhanced psf (see Fig. 6E). The images obtained with a monolithic Au-coated tip (thickness of $10 \mathrm{~nm}$ ), on the other hand, showed a diffraction-limited psf with a sharp dip (see Fig. 6F) indicating tip-induced fluorescence quenching instead of tip-enhancement (compare Fig. 6E and $\mathrm{F}$ ).

As recently confirmed experimentally by Maouli et al. ${ }^{53}$ the tip with isolated nanostructures show better local field enhancement than the tips monolithically coated with gold or silver. This difference originates from the fact that the extended cone-structures associated with metal-coated tips are largely off-resonant with the excitation light, whereas the isolated nanostructures are fully resonant (dipolar plasmons) with the visible radiation. The ideal cone-shaped TERS tips should in theory provide extreme field enhancement, yet in reality such geometry is hardly achieved in typical experiments. In particular for the TEF measurement, there exists surfaceinduced quenching competing against the fluorescence enhancement caused by the local field enhancement. The quenching rate for the nanostar and coated tips will be similar. For the monolithic tip, the field enhancement is insufficient to overcome the fluorescence quenching, whereas for the nanostar tip, the enhancement is sufficient. This is why we observe a dip (quenching) in the TEF images obtained with the coated tip (Fig. 6F).
We believe that there still exists room for further improvement of the nanostar tip and their application in tip-enhanced spectroscopy. The dipolar plasmon of the nanostar is not optimally resonant with the excitation wavelength. With further refinement of the structure or use of more appropriate excitation wavelength, we will be able to optimize the field enhancement. The mechanical stability of the nanostar probe may be increased by treating it chemically and/or thermally during the preparation processes. Besides the plasmonic properties of the tip, the properties of the sample (molecules and substrate) will influence the spectroscopic enhancement. For the TERS measurement, the enhancement will be influenced by the tip-sample distance and the local morphology of the substrate (note that our substrate has a finite roughness). For the TEF measurement, we use the PMMA matrix to immobilize the target molecule. The finite thickness of the PMMA layer, as well as the random orientation of the molecule, will cause the variation in fluorescence enhancement through the variation in the tip-molecule distance. Currently, we are exploring ways to improve such variabilities of sample/substrate, as well as plasmonic properties.

\section{Conclusions}

The synthetic procedure we developed for the nanostar probe and its performance for TERS and TEF showed that the 
current approach is viable for TENOM, although challenges remain. The synthetic strategy, picking up a seed particle followed by growing the nanoparticle in situ, allowed us to make nanostar tips with high yield (67\%) and with reasonable mechanical stability. The majority of such probes showed local field enhancements that were useful for TERS and TEF measurements. At the same time, however, these tips showed large tipto-tip variations in nano-optical properties, which we believe originated from the dispersion of the nanostar shape and the variation in spike orientation. The structural imperfections as well as the resonance characteristics because the current excitation wavelength of $532 \mathrm{~nm}$ was not resonant with the nanostar plasmon resonance (see Fig. 1), may have contributed to the marginal spatial resolution $(\sim 100 \mathrm{~nm})$ and the field enhancement observed in TERS and TEF. The synthetic procedure we developed is a generic method that is not restricted to nanostar formation. By carefully tuning the growth steps, the plasmonic properties of nanostars can be further optimized, or different shapes of nanoparticles (such as octahedra or cubes) can be grown at the end of the tip. Such size and shape fine-tuning will help improve the performance of the tip for improved mechanical stability, signal enhancement, and spatial resolution in TERS and TEF. Above all, the reliable yield of the nanostar probe fabrication is one merit of the approach, but the effectiveness of the TENOM probes is still limited. Extended investigation of such probes promises deeper understanding of the optical phenomena and fabrication of more effective probes.

\section{Acknowledgements}

J. W. P. acknowledges the Brain Research Program (2015030964) of the National Research Foundation (NRF) of Korea and the Converging Research Center Program (2015K000211) of the Ministry of Science, ICT, and Future Planning, Korea, and Z. H. K. and N. K. were supported by BioNano Health-Guard Research Center funded by the Ministry of Science, ICT \& Future Planning (MSIP) of Korea as Global Frontier Project (H-GUARD_2013M3A6B2078947), and the Research Resettlement Fund for the New Faculty of SNU.

\section{References}

1 A. Hartschuh, Angew. Chem., Int. Ed., 2008, 47, 8178-8191.

2 E. Bailo and V. Deckert, Chem. Soc. Rev., 2008, 37, 921-930.

3 B. Pettinger, P. Schambach, C. J. Villagomez and N. Scott, Annu. Rev. Phys. Chem., 2012, 63, 379-399.

4 B. S. Yeo, J. Stadler, T. Schmid, R. Zenobi and W. H. Zhang, Chem. Phys. Lett., 2009, 472, 1-13.

5 E. Betzig and J. K. Trautman, Science, 1992, 257, 189-194.

6 L. Novotny and N. van Hulst, Nat. Photonics, 2011, 5, 83-90.

7 B. Huang, M. Bates and X. W. Zhuang, Annu. Rev. Biochem., 2009, 78, 993-1016.

8 M. Fernandez-Suarez and A. Y. Ting, Nat. Rev. Mol. Cell Biol., 2008, 9, 929-943.
9 W. Bao, M. Melli, N. Caselli, F. Riboli, D. S. Wiersma, M. Staffaroni, H. Choo, D. F. Ogletree, S. Aloni, J. Bokor, S. Cabrini, F. Intonti, M. B. Salmeron, E. Yablonovitch, P. J. Schuck and A. Weber-Bargioni, Science, 2012, 338, 1317-1321.

10 P. Bharadwaj, P. Anger and L. Novotny, Nanotechnology, 2007, 18, 044017.

11 A. Singh, G. Calbris and N. F. van Hulst, Nano Lett., 2014, 14, 4715-4723.

12 R. M. Stockle, Y. D. Suh, V. Deckert and R. Zenobi, Chem. Phys. Lett., 2000, 318, 131-136.

13 N. Anderson, A. Hartschuh, S. Cronin and L. Novotny, J. Am. Chem. Soc., 2005, 127, 2533-2537.

14 T. Ichimura, S. Fujii, P. Verma, T. Yano, Y. Inouye and S. Kawata, Phys. Rev. Lett., 2009, 102, 186101.

15 R. Zhang, Y. Zhang, Z. C. Dong, S. Jiang, C. Zhang, L. G. Chen, L. Zhang, Y. Liao, J. Aizpurua, Y. Luo, J. L. Yang and J. G. Hou, Nature, 2013, 498, 82-86.

16 N. Mauser and A. Hartschuh, Chem. Soc. Rev., 2014, 43, 1248-1262.

17 T. W. Johnson, Z. J. Lapin, R. Beams, N. C. Lindquist, S. G. Rodrigo, L. Novotny and S. H. Oh, ACS Nano, 2012, 6, 9168-9174.

18 E. M. van Schrojenstein Lantman, T. Deckert-Gaudig, A. J. G. Mank, V. Deckert and B. M. Weckhuysen, Nat. Nanotechnol., 2012, 7, 583-586.

19 J. Stadler, T. Schmid and R. Zenobi, Nano Lett., 2010, 10, 4514-4520.

20 B. Ren, G. Picardi and B. Pettinger, Rev. Sci. Instrum., 2004, 75, 837-841.

21 M. Mivelle, T. S. van Zanten, L. Neumann, N. F. van Hulst and M. F. Garcia-Parajo, Nano Lett., 2012, 12, 5972-5978.

22 T. H. Taminiau, R. J. Moerland, F. B. Segerink, L. Kuipers and N. F. van Hulst, Nano Lett., 2007, 7, 28-33.

23 S. Berweger, J. M. Atkin, R. L. Olmon and M. B. Raschke, J. Phys. Chem. Lett., 2012, 3, 945-952.

24 S. Kuhn, U. Hakanson, L. Rogobete and V. Sandoghdar, Phys. Rev. Lett., 2006, 97, 017402.

25 T. Kalkbrenner, U. Hakanson, A. Schadle, S. Burger, C. Henkel and V. Sandoghdar, Phys. Rev. Lett., 2005, 95, 200801.

26 H. Eghlidi, K. G. Lee, X. W. Chen, S. Gotzinger and V. Sandoghdar, Nano Lett., 2009, 9, 4007-4011.

27 J. G. Zhang, Y. Gao, R. A. Alvarez-Puebla, J. M. Buriak and H. Fenniri, Adv. Mater., 2006, 18, 3233-3237.

28 L. C. Cheng, J. H. Huang, H. M. Chen, T. C. Lai, K. Y. Yang, R. S. Liu, M. Hsiao, C. H. Chen, L. J. Her and D. P. Tsai, J. Mater. Chem., 2012, 22, 2244-2253.

29 C. L. Nehl, H. W. Liao and J. H. Hafner, Nano Lett., 2006, 6, 683-688.

30 H. K. Yuan, C. G. Khoury, H. Hwang, C. M. Wilson, G. A. Grant and T. Vo-Dinh, Nanotechnology, 2012, 23, 075102.

31 P. P. Pompa, L. Martiradonna, A. Della Torre, F. Della Sala, L. Manna, M. De Vittorio, F. Calabi, R. Cingolani and R. Rinaldi, Nat. Nanotechnol., 2006, 1, 126-130. 
32 K. Aslan, M. Wu, J. R. Lakowicz and C. D. Geddes, J. Am. Chem. Soc., 2007, 129, 1524-1525.

33 G. M. Akselrod, C. Argyropoulos, T. B. Hoang, C. Ciraci, C. Fang, J. N. Huang, D. R. Smith and M. H. Mikkelsen, Nat. Photonics, 2014, 8, 835-840.

34 D. Kim, N. K. Chung, J. S. Kim and J. W. Park, Soft Matter, 2010, 6, 3979-3984.

35 D. Kim, N. K. Chung, S. Allen, S. J. B. Tendler and J. W. Park, ACS Nano, 2012, 6, 241-248.

36 D. Kim and O. Sahin, Nat. Nanotechnol., 2015, 10, 264-269.

37 Y. J. Jung, B. J. Hong, W. Zhang, S. J. B. Tendler, P. M. Williams, S. Allen and J. W. Park, J. Am. Chem. Soc., 2007, 129, 9349-9355.

38 Lumerical Solutions, http://www.lumerical.com/.

39 K. Kumagai and A. Ishida, Bull. Chem. Soc. Jpn., 2014, 87, 780-791.

40 K. Liu, Y. Song, W. Feng, N. N. Liu, W. K. Zhang and X. Zhang, J. Am. Chem. Soc., 2011, 133, 3226-3229.

41 P. Hinterdorfer and Y. F. Dufrene, Nat. Methods, 2006, 3, 347-355.

42 C. J. Orendorff and C. J. Murphy, J. Phys. Chem. B, 2006, 110, 3990-3994.

43 M. Z. Liu and P. Guyot-Sionnest, J. Phys. Chem. B, 2005, 109, 22192-22200.
44 M. Grzelczak, J. Perez-Juste, P. Mulvaney and L. M. Liz-Marzan, Chem. Soc. Rev., 2008, 37, 17831791.

45 R. Michalitsch, B. J. Palmer and P. E. Laibinis, Langmuir, 2000, 16, 6533-6540.

46 T. K. Sau and C. J. Murphy, J. Am. Chem. Soc., 2004, 126, 8648-8649.

47 L. Lu, A. Kobayashi, K. Tawa and Y. Ozaki, Chem. Mater., 2006, 18, 4894-4901.

48 H. Ishitobi, I. Nakamura, T. A. Kobayashi, N. Hayazawa, Z. Sekkat, S. Kawata and Y. Inouye, ACS Photonics, 2014, 1, 190-197.

49 A. M. Chizhik, R. Jager, A. I. Chizhik, S. Bar, H. G. Mack, M. Sackrow, C. Stanciu, A. Lyubimtsev, M. Hanack and A. J. Meixner, Phys. Chem. Chem. Phys., 2011, 13, 17221733.

50 M. Mivelle, T. S. van Zanten and M. F. Garcia-Parajo, Nano Lett., 2014, 14, 4895-4900.

51 P. Anger, P. Bharadwaj and L. Novotny, Phys. Rev. Lett., 2006, 96, 113002-113001.

52 S. Kuhn and V. Sandoghdar, Appl. Phys. B, 2006, 84, 211217.

53 I. Maouli, A. Taguchi, Y. Saito, S. Kawata and P. Verma, Appl. Phys. Express, 2015, 8, 032401. 